\title{
Ludificación (gamification) y exámenes on-line como elemento dinamizador y motivador del estudio
}

\author{
Fernando Cardona ${ }^{\text {a, }}$, Lorena Atarés-Huerta ${ }^{a}$ \\ ${ }^{a}$ Departamento de Tecnología de los Alimentos. Escuela Técnica Superior de Ingeniería Agronómica y del Medio \\ Natural. Universitat Politècnica de València. \\ ${ }^{\mathrm{b}}$ Unitat de Genètica Molecular. Instituto de Biomedicina de Valencia. Consejo Superior de Investigaciones \\ Científicas.
}

\begin{abstract}
Resumen
La ludificación (gamification) se utiliza con frecuencia como elemento motivador y dinamizador en el aula, permitiendo introducir actividades recreativas durante la lección magistral. En general es bien aceptada por los alumnos, y permite introducir y asentar conceptos complejos de manera amena.
\end{abstract}

En este trabajo se utilizó Socrative para realizar los cuestionarios de repaso de los conceptos teóricos, que se realizaban ya anteriormente en el aula, dando además el feedback al estudiante inmediatamente (proporcionándole la respuesta correcta, una pequeña explicación, y la calificación obtenida). Además, el examen de teoría se realizó on-line utilizando la herramienta correspondiente en la plataforma de la Universitat Politècnica de València (Poliformat).

Los resultados académicos muestran que las calificaciones mejoran en los estudiantes que realizan los cuestionarios con Socrative en clase, probablemente debido a que la evaluación formativa y gamificada mejora la asimilación de los conceptos teóricos. Sin embargo, los resultados no son estadísticamente significativos.

Las encuestas realizadas muestran además que a los alumnos en general les gustan estas dos actividades, están de acuerdo con su diseño, y perciben que contribuyen de forma importante a su aprendizaje y sus calificaciones.

Sin embargo, se observó que son necesarias algunas modificaciones para facilitar la aplicación de esta mejora por parte del profesorado. En cuanto al uso de Socrative, una dificultad observada es que la corrección de los cuestionarios resulta demasiado tediosa para el profesor en la versión gratuita de Socrative. Además, los alumnos plantean la posibilidad de hacer los cuestionarios incluso fuera de los horarios de clase, por lo que parece 
apropiado introducir ciertas modificaciones. En cuanto a los exámenes online, la principal dificultad reside en impedir que los alumnos copien de los compañeros o de la web, por lo que se han buscado herramientas para solventarlo. Basándonos en esta experiencia se proponen algunas modificaciones de estas actividades.

Palabras clave: Ludificación, Gamification, Socrative, Examen on-line, Motivación, Dinamización, Evaluación formativa.

\section{Introducción}

La ludificación es la aplicación de mecánicas de juego a situaciones que en principio no son lúdicas, como puede ser el proceso enseñanza-aprendizaje (Deterding, 2011) y ha demostrado ser útil aumentando la motivación y reduciendo el estrés (Hanus y Fox, 2015), debido fundamentalmente a que es un proceso divertido y suele proporcionar un feedback inmediato (Llorens-largo et al., 2016). Este tipo de actividades también facilitan el aprendizaje por el propio carácter lúdico de la actividad, que reconforta al alumno, incentivándolo en el proceso (Koster y Wright, 2004). También resulta útil, como cualquier actividad y en especial las de carácter lúdico, para interrumpir temporalmente la lección magistral rompiendo así la monotonía y manteniendo al alumno activo, que de otra manera acabaría desconectando (Prieto-Martín et al., 2014).

Dado que actualmente casi el 100\% del alumnado dispone de dispositivos móviles de pantalla táctil (tabletas, móviles y ordenadores híbridos), parece apropiado introducirlos en la dinámica del aula para realizar las actividades, más aún si son dispositivos a los que dedican mucho tiempo y atención, como es el caso del teléfono móvil. Sin embargo, esta mejora en la motivación mediante el uso de dispositivos digitales no siempre se refleja en una mejora del proceso de aprendizaje de todas las competencias. Por ejemplo, se ha relacionado su uso con el empeoramiento de las habilidades de escritura, y en el aprendizaje de cuestiones teóricas cuando se evalúan mediante respuesta larga abierta (Wollscheid et al., 2016; Wakefield et al., 2018). Es por ello que es necesario un estudio piloto antes de introducirlas en las actividades del aula.

Por otro lado, los exámenes on-line han demostrado disminuir la ansiedad y estrés del alumno, lo que lleva a una mejor evaluación del aprendizaje (Veenman et al., 2005). También, proporcionan un feedback inmediato de los resultados, lo que resulta en una mejora de la evaluación formativa (Epstein et al., 2002). 
La hipótesis de trabajo de este proyecto es que la introducción de dispositivos digitales en las actividades del aula y en la evaluación puede mejorar el rendimiento académico. En concreto se utilizaron dispositivos táctiles (fundamentalmente el teléfono móvil) en la realización de los cuestionarios de repaso de la teoría, que hasta ahora venían haciéndose en papel, utilizando el programa Socrative (https://www.socrative.com/), de forma que los alumnos resuelven los cuestionarios en el aula, incentivándolos además con una pequeña subida en la nota de teoría (hasta 0,3 puntos sobre la nota obtenida en el examen), sin variar el máximo que puede obtenerse (3 sobre 10). También se introdujo la realización del examen de teoría basado en estos cuestionarios ludificados, realizando el examen on-line en un aula de informática de la Universitat Politècnica de València mediante la correspondiente plataforma educativa (Poliformat). Casi la totalidad de los alumnos eligió esta modalidad de examen, lo que demuestra su elevada aceptación. Sin embargo, hubo algunos problemas de implementación, por lo que se proponen algunas modificaciones para implementar esta actividad.

\section{Objetivos}

El objetivo general de este trabajo es determinar si la introducción de la gamificación mediante el uso de dispositivos digitales tiene un efecto positivo sobre el proceso de enseñanza-aprendizaje. Para ello, este objetivo general se subdivide en tres objetivos parciales:

2.1-Evaluar el efecto en la calificación final y la aceptación del uso de Socrative para la realización de los cuestionarios de teoría de la UD2 de la asignatura "Fundamentos Químicos para Ciencia y Tecnología de Alimentos (CTA)", que venían realizándose hasta la fecha en papel.

2.2-Evaluar el efecto sobre la calificación final y la aceptación de la introducción de exámenes de la parte teórica on-line, utilizando las preguntas de los cuestionarios de Socrative.

2.3-Plantear las modificaciones necesarias de ambas actividades para implantarlas en la evaluación, en caso de tener un efecto positivo y una aceptación alta. 


\section{Desarrollo}

Esta experiencia se llevó a cabo con alumnos de primer curso del grado de CTA, de la Universitat Politècnica de València (UPV). La asignatura es anual de 12 créditos repartidos en tres Unidad Didácticas (UD). Este proyecto se llevó a cabo en la UD2 (Química Física).

Durante el curso académico 2017-2018 se implantó en el aula el uso de Socrative para realizar los cuestionarios de repaso de la parte teórica de la UD2, que hasta el momento se realizaba en papel y se corregía luego en la pizarra. Además, se introdujo la posibilidad de realizar el examen de teoría on-line una semana antes de la prueba final, utilizando la herramienta exámenes de Poliformat, siendo este examen eliminatorio, de forma que en caso de aprobarlo para el examen final solo se realiza la parte de problemas. El examen consiste en una batería de 30 preguntas aleatorizadas, limitando el acceso a las IPs del aula del examen, y la corrección del examen se envía automáticamente por e-mail al finalizarlo, junto con la calificación.

Con una encuesta posterior se midió la opinión de los alumnos sobre estas metodologías, así como los beneficios que perciben. Para realizar la encuesta y analizar los resultados se utilizó la herramienta correspondiente de Google Drive (Cuestionarios de Google). El efecto sobre la nota final alcanzada se evaluó utilizando la herramienta Excel de Microsoft Office.

De los 91 alumnos examinados, 63 utilizaron Socrative con cierta asiduidad en clase, de los cuales 45 rellenaron la encuesta. De estos 91, 88 realizaron el examen de teoría on-line, de los cuales 45 rellenaron la correspondiente encuesta.

\section{Resultados y discusión}

\subsection{Opinión de los alumnos}

\subsubsection{Uso de Socrative}

De los alumnos encuestados (45), al 84,4\% le ha gustado usar Socrative y le ha parecido útil (Fig. 1), frente a un $8,9 \%$ a los que no. En cuanto al diseño de los cuestionarios (Fig. 2), un $93,3 \%$ opina que es bueno o muy bueno, por lo que no parecen necesarias modificaciones de los mismos. En lo relativo a la contribución al aprendizaje de los conceptos, un $66,6 \%$ opina que es alto o muy alto (Fig. 3). Además, un $86,6 \%$ piensa que la 
contribución a la mejora de la calificación es adecuada o alta (Fig. 4), por lo que no se plantea realizar modificaciones en ese sentido.

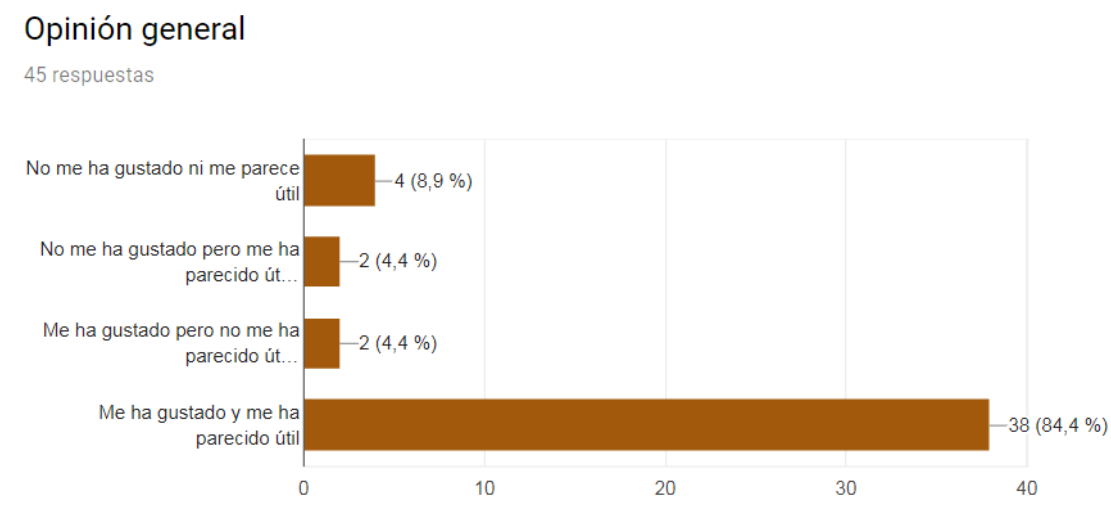

Fig. 1. Opinión general de los alumnos sobre el uso de Socrative. Se muestra el porcentaje de alumnos que ha contestado cada opción en la encuesta. Se observa que a la mayoría de los alumnos (84.4\%) les gusta la actividad y les parece útil. Fuente: Elaboración propia

En general, un $84,4 \%$ de los encuestados recomienda su uso (Fig. 5), por lo que puede concluirse que existe una buena aceptación de la metodología utilizada.

Dos mejoras han sido propuestas por los alumnos encuestados:

i) Subir las respuestas correctas a Poliformat (plataforma educativa de la UPV)

ii) Habilitar la posibilidad de realizar la actividad fuera del aula

La primera modificación propuesta se desestima por ir en contra del objetivo mismo de la actividad, que es realizar en el aula los cuestionarios para que el, interaccione con el profesor en clase y repase los contenidos conforme se van explicando. Sin embargo, sí parece apropiada, aunque con modificaciones, la segunda propuesta. Para cursos posteriores se plantea introducir los cuestionarios como exámenes on-line calificables (hasta un punto de la nota final) que se realizarán fuera del aula, mientras que el uso de Socrative quedaría restringido al aula, pero sin efecto en las calificaciones. 


\section{Cuestionarios (diseño de las preguntas y adecuación al examen)}

45 respuestas

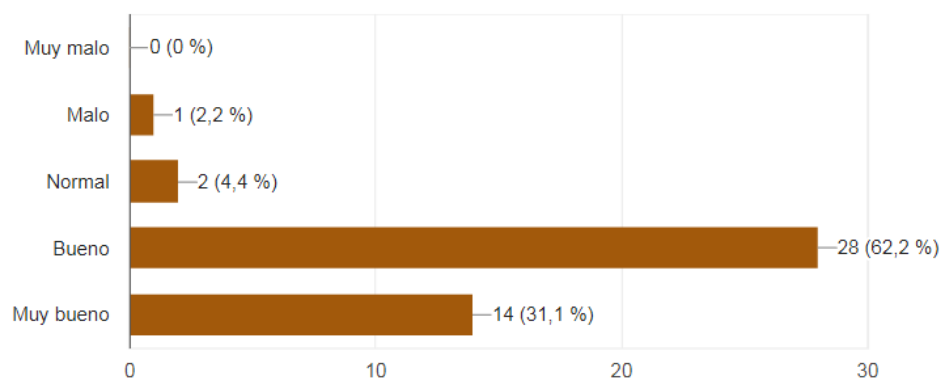

Fig. 2. Opinión de los alumnos sobre los cuestionarios utilizados para repasar la teoría en el aula. Se muestra el porcentaje de alumnos que ha contestado cada opción en la encuesta. Se observa que a la mayoría de los alumnos $(62.2+31.1=73.3 \%)$ el diseño de las preguntas y la adecuación al examen les parecen buenos o muy buenos. Fuente: Elaboración propia

\section{Contribución al aprendizaje de los conceptos}

45 respuestas

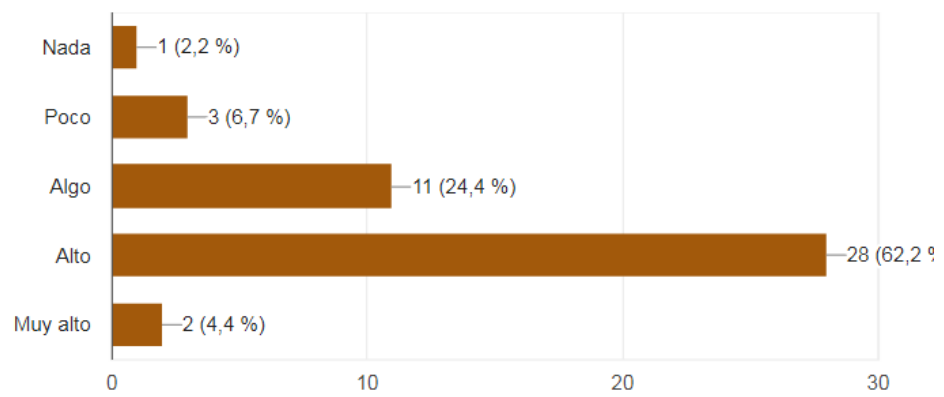

Fig. 3. Opinión de los alumnos sobre la contribución del examen on-line al aprendizaje en la asignatura. Se muestra el porcentaje de alumnos que ha contestado cada opción en la encuesta. Se observa que la mayoría de los alumnos $(62.2+4.4=66.6 \%)$ opina que la actividad contribuye al aprendizaje de los conceptos.

Fuente: Elaboración propia 
Importancia del efecto sobre la nota final

45 respuestas

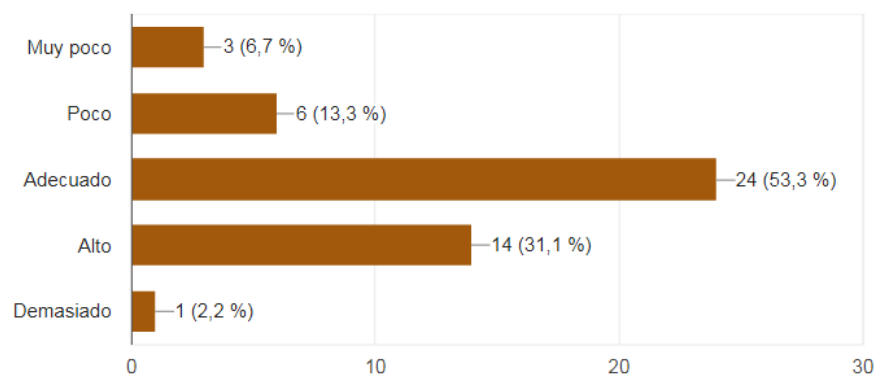

Fig. 4. Opinión de los alumnos sobre la importancia en la nota final del examen on-line. Se muestra el porcentaje de alumnos que ha contestado cada opción en la encuesta. Se observa que la mayoría de los alumnos $(53.3+31.1=64.4 \%)$ opina que el efecto de la actividad sobre la nota final es adecuado o alto sin ser excesivo. Fuente: Elaboración propia

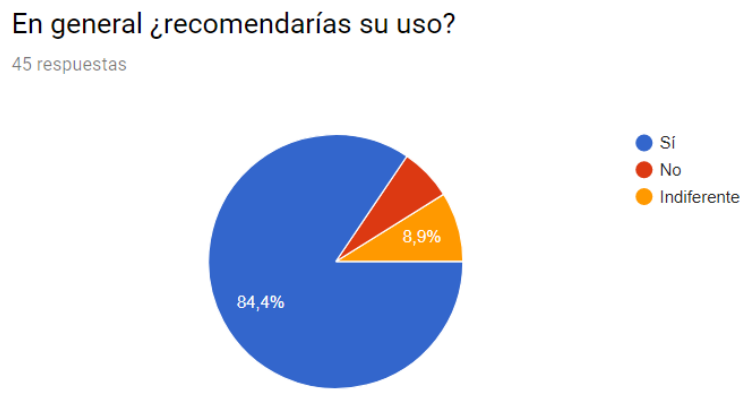

Fig. 5. Recomendación general de los alumnos sobre la realización del examen on-line. Se muestra el porcentaje de alumnos que ha contestado cada opción en la encuesta. Se observa que la mayoría de los alumnos (84.4\%) recomiendan su uso. Fuente: Elaboración propia

\subsubsection{Examen on-line}

De los 91 estudiantes matriculados, 87 realizaron el examen on-line, y de éstos, 45 realizaron la encuesta planteada sobre el mismo. De esos 45 estudiantes, 25(55,5\%) prefieren hacerlo on-line, y solo a 1 no le gusta en absoluto (Fig. 6), por lo que podemos concluir que tiene una buena aceptación.

En cuanto al contenido del examen (calidad de las preguntas, correspondencia con lo trabajado en el aula, número y tipo de preguntas y tiempo para contestar), el 83,5\% está de acuerdo, mientras ningún estudiante está en desacuerdo (Fig. 7), por lo que el diseño del examen parece correcto y no necesita modificaciones sustanciales. 
En lo referente al feedback y rapidez en conocer la nota, el $84 \%$ está de acuerdo (Fig.8), por lo que también parece un método adecuado para la evaluación formativa.

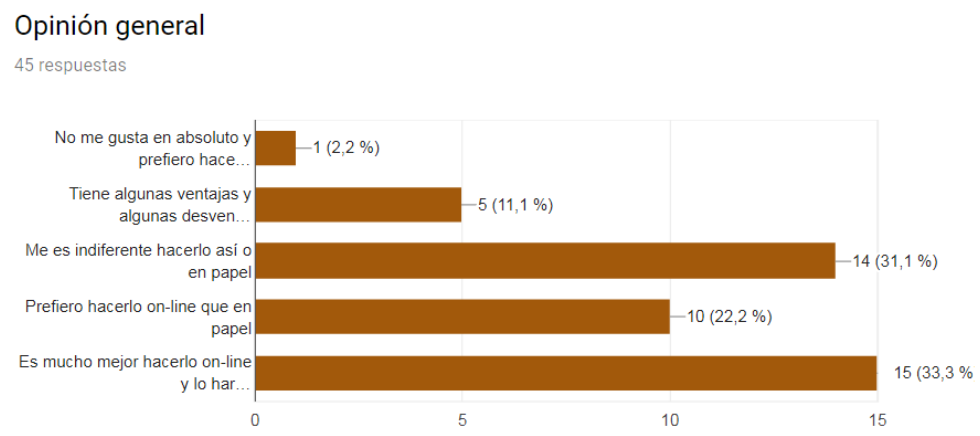

Fig. 6. Opinión general de los alumnos sobre el examen on-line. Se muestra el porcentaje de alumnos que ha contestado cada opción en la encuesta. Se observa que la mayoría de los alumnos $(22.2+33.3=55.5 \%)$ prefiere hacer el examen on-line. Además, al 31.1\% le es indiferente, por lo que solo un $13.3 \%$ le encuentran inconvenientes. Fuente: Elaboración propia.
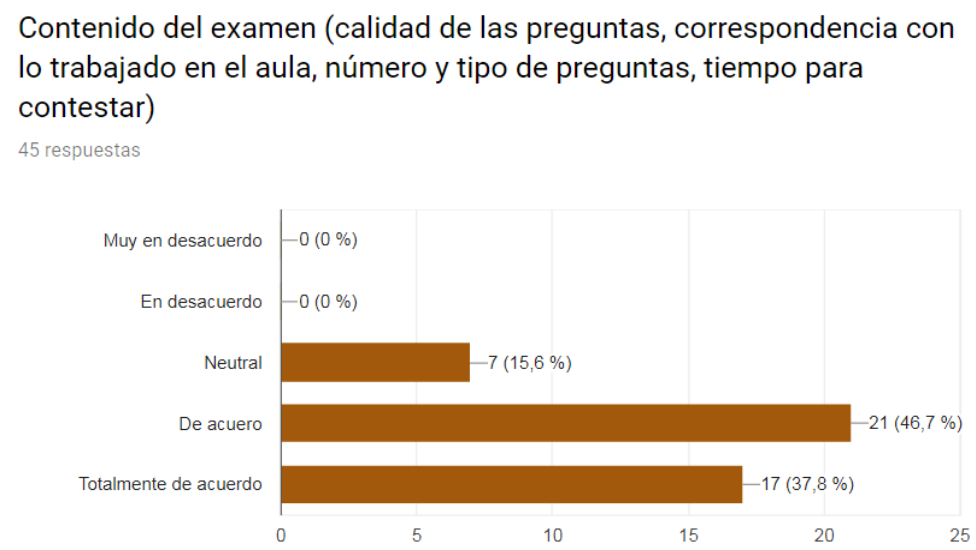

Fig. 7. Opinión de los estudiantes sobre el contenido del examen on-line. Se muestra el porcentaje de alumnos que ha contestado cada opción en la encuesta. Se observa que la gran mayoría de los alumnos $(46.7+37.8=83.5 \%)$ están de acuerdo con el contenido del examen, y ninguno está en desacuerdo. Fuente: Elaboración propia. 
Evaluación formativa (feedback con respuestas correctas, errores cometidos) y rapidez en el conocimiento de la nota

44 respuestas

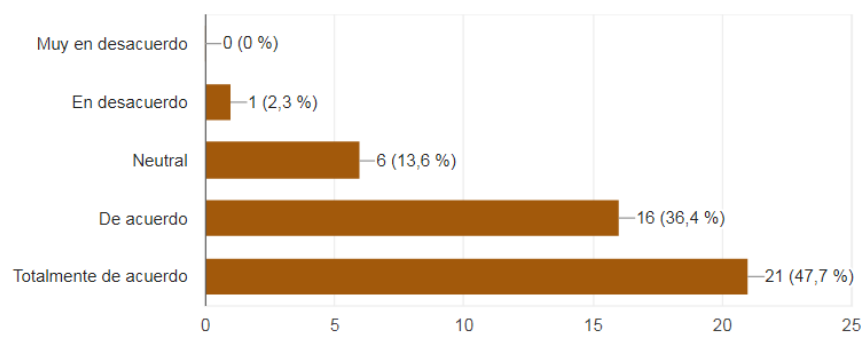

Fig. 8. Opinión de los estudiantes sobre la evaluación formativa del examen on-line. Se muestra el porcentaje de alumnos que ha contestado cada opción en la encuesta. Se observa que la mayoría de los alumnos $(36.4+47.7=$ $84.1 \%)$ les gusta el tipo de examen en lo que respecta a evaluación formativa, y solo un alumno (2.3\%) está en desacuerdo. Fuente: Elaboración propia.

Por último, cabe destacar que el $82 \%$ de los encuestados recomienda hacer el examen de teoría mediante esta modalidad (Fig.9), por lo que consideramos que tiene una elevada aceptación.

En general ¿recomendaría hacer el examen on-line?

45 respuestas
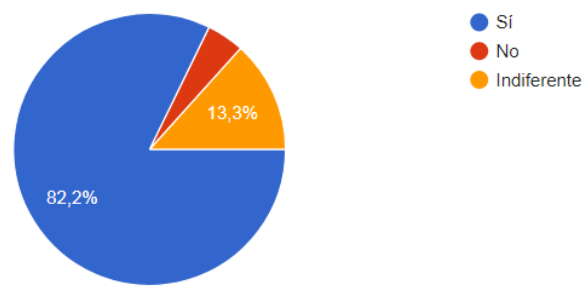

Fig. 9. Recomendación general de los estudiantes sobre el examen on-line. Se muestra el porcentaje de alumnos que ha contestado cada opción en la encuesta. Se observa que la mayoría de los alumnos (82.2\%) recomienda hacer el examen de esta manera. Fuente: Elaboración propia.

\subsection{Efectos de la ludificación en las calificaciones de los alumnos}

El efecto de realizar el examen on-line en la nota final no pudo ser evaluado por no tener dos grupos para comparar, debido a que prácticamente todos los alumnos eligieron realizar el examen on-line. 
Sí que se pudo medir la influencia del uso de Socrative para realizar los cuestionarios, ya que no todos los estudiantes participaron en esta actividad, y algunos lo hicieron muy esporádicamente. Para este análisis, se incluyeron en el grupo de estudiantes que habían participado en la actividad, aquellos que alcanzaron al menos 0,1 puntos sobre 0,3 , para no incluir a los que habían participado solo en alguno de los cuestionarios.

Los resultados obtenidos (Fig. 10) muestran cierta tendencia a la mejora en la parte del examen de TA (media de 2,53 frente a 2,43), y para la nota global del examen $(6,16$ frente a 5,62 ), aunque estos resultados no son estadísticamente significativos ( $p>0,05$, ver panel inferior). Si son significativas las diferencias en las notas finales $(6,3$ frente a 5,2, $p=0,001)$, pero ese dato incluye el incremento en la calificación debida a la realización de la actividad, por lo que con estos datos no puede concluirse que mejore el aprendizaje de la TA ni de la asignatura en general. Sin embargo, dado que se observa cierta tendencia positiva a la mejora, es posible que en una muestra poblacional diferente los resultados sean estadísticamente significativos.

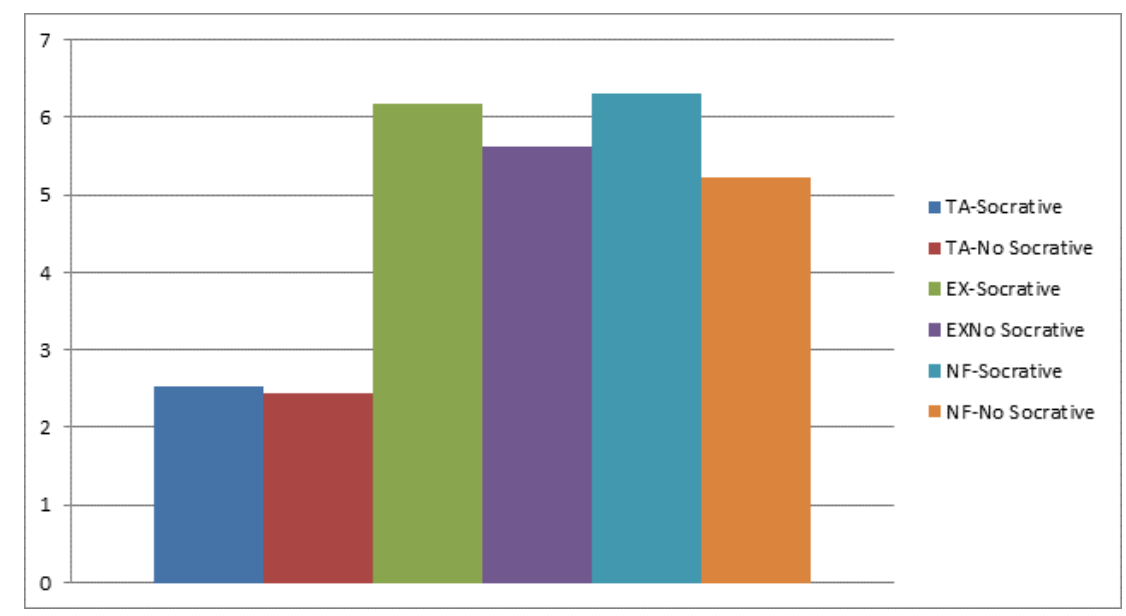

\begin{tabular}{|c|c|c|c|c|c|c|c|c|}
\hline$T A$ & Socrative & No Socrative & NOTA EXAMEN & \begin{tabular}{l|} 
Socrative \\
\end{tabular} & No Socrative & NOTA FINAL & Socrative & No Socrative \\
\hline Media & 2,53757812 & 2,4325 & Media & 6,16600781 & 5,62518519 & Media & 6,30697656 & 5,21135185 \\
\hline Varianza & 0,16353591 & 0,09766587 & Varianza & 3,68093072 & 2,42379323 & Varianza & 3,5168613 & 1,72898511 \\
\hline Observaciones & 64 & 27 & Observaciones & 64 & 27 & Observaciones & 64 & 27 \\
\hline Diferencia hipotética de las medias & & & Diferencia hipotética de las medias & 0 & & Diferencia hipotética de las medias & 0 & \\
\hline Grados de libertad & 63 & & Grados de libertad & 60 & & Grados de libertad & 69 & \\
\hline Estadistico $t$ & 1,33746266 & & Estadistico $t$ & 1,40921122 & & Estadistico $t$ & 3,17622494 & \\
\hline$P(T<=t)$ una cola & 0,09294018 & & $P(T<=t)$ una cola & 0,08196733 & & $P(T<=t)$ una cola & 0,00111583 & \\
\hline Valor critico de $t$ (una cola) & 1,66940222 & & Valor critico de t (una cola) & 1,67064887 & & Valor crítico de $t$ (una cola) & 1,66723855 & \\
\hline $\mathrm{P}(\mathrm{T}<=\mathrm{t})$ dos colas & 0,18588035 & & $P(T<=t)$ dos colas & 0,16393466 & & $P(T<=t)$ dos colas & 0,00223166 & \\
\hline Valor critico de $\mathrm{t}$ (dos colas) & 1,99834052 & & Valor critico de $\mathrm{t}$ (dos colas) & 2,0002978 & & Valor critico de t (dos colas) & 1,99494539 & \\
\hline
\end{tabular}

Fig. 10. Efecto del uso de Socrative para realizar los cuestionarios de teoría sobre las calificaciones obtenidas. Se muestra en el diagrama de barras las calificaciones obtenidas para cada grupo de alumnos. Los resultados muestran que, aunque el efecto en la nota final es significativo (ver tabla inferior, $\mathrm{p}$-valor $<0,05$ ), es debido al incremento de la nota por la actividad. No existen diferencias significativas en las calificaciones obtenidas por los alumnos en los exámenes. $\mathrm{TA}=$ nota examen teoría de aula. $\mathrm{EX}=$ nota total examen. $\mathrm{NF}=$ nota final. Fuente: Elaboración propia. 


\section{Conclusiones}

Según los resultados obtenidos en este trabajo, la ludificación de los cuestionarios mediante Socrative:

1-Tiene buena aceptación por los alumnos. Además, opinan que está realizado con unos cuestionarios adecuados, contribuye al aprendizaje de los conceptos y la aportación a la calificación es adecuada.

2-Aunque se observa cierta tendencia positiva, no parece contribuir significativamente a mejorar el aprendizaje medido como mejora en las calificaciones.

En estas condiciones, la introducción del examen de TA on-line:

3- Tiene buena aceptación por los alumnos y lo prefieren al examen escrito. También opinan que el contenido del examen es adecuado, se ajusta a los contenidos trabajados en el aula y contribuye adecuadamente a la evaluación formativa.

\section{Modificaciones propuestas y perspectivas}

A la vista de los resultados, parece interesante seguir con las metodologías iniciadas. Aunque la mejora en el aprendizaje nos sea significativa se observa cierta tendencia positiva, y son actividades que gustan a los alumnos. Sin embargo, se han detectado algunos puntos de mejora, no en las actividades en sí mismas si no en su implementación:

-Para la ludificación se hace necesario mejorar el sistema de corrección de la actividad. Con la versión gratuita de Socrative se almacenan los cuestionarios con el nombre que pone el alumno, que puede ser diferente cada vez. Los profesores deben luego recopilar los nombres y la puntuación de cada cuestionario, lo que se convierte en una tarea bastante ardua. Además, un alumno podría hacer el cuestionario en nombre de otro o esperar a que el compañero (que puede firmar con un nombre falso) conteste y ver así la respuesta correcta. Es por ello que se propone usar la versión de pago de Socrative, que sí pide nombre real y almacena las puntuaciones, permitiendo entrar solo si se está registrado, con un login/password que almacena las respuestas. Otra opción, si no se dispone de la versión de pago, es utilizar Socrative para "ensayar" el cuestionario, y luego realizar la actividad calificable (en el aula o fuera de ella) en la plataforma educativa (Poliformat), que es personal y almacena las puntuaciones, de forma que luego puedes promediar y procesar las calificaciones de manera sencilla. Cabe destacar que algunos alumnos han sugerido poder 
realizar la actividad fuera del aula si no asisten a clase, por lo que se plantea esta opción como futura mejora de la actividad, puntuando un porcentaje de la nota final. Pensamos que de esta forma se obliga al alumno a estudiar casi "al día" la teoría impartida.

-En cuanto al examen on-line, el principal problema es impedir la copia durante el mismo, lo que se ha solucionado contactando con el departamento correspondiente de la UPV (ASIC). El próximo curso académico, además de aleatorizar el orden de las preguntas, éstas podrán ser diferentes para cada alumno (al menos en parte) y el alumno solo podrá acceder al examen (no a Poliformat ni a internet en general) en esa aula.

\section{Referencias}

Deterding, S., Icart, M., Nacke, L. E., O’Hara, J. \& Dixon, D. (2011). Gamification: Using Game Design Elements in Non-Gaming Contexts. Proceedings of CHI EA, ACM.

Epstein, M. L., Amber, D., Lazarus, A. D., Calvano, T. B., Matthews, K. A., Hendel, R. A., Epstein, B. B. \& Brosvic, G.M. (2002) Immediate Feedback Assessment Technique Promotes Learning and Corrects Inaccurate first Responses. The Psychological Record. 52(2): 187-201

Hanus, M. D. \& Fox, J. (2015). Assessing the effects of gamification in the classroom: A longitudinal study on intrinsic motivation, social comparison, satisfaction, effort, and academic performance. Computers \& Education. 80:152-161.

Koster, R. \& Wright, W. (2004). A theory of fun. New York: Paraglyph Press.

Lorens-Largo, F., Villagrá-Arnedo, C. J., Gallego-Durán, F. J., Satorre-Cuerda, R., Compañ-Rosique, P. \& Molina-Carmona, R. (2016) LudifyME: An Adaptive Learning Model Based on Gamification. Formative Assessment, Learning Data Analytics and Gamification: 245-269

Prieto Martin, A., Díaz Martín, D., Montserrat Sanz, J. \& Reyes Martín, E. (2014). Experiencias de aplicación de estrategias de gamificación a entornos de aprendizaje universitario. ReVisión 7: 2743.

Wakefield, J., Frawley, J. K., Tyler , J. \& Dyson, L. E. (2018) The impact of an iPad-supported annotation and sharing technology on university students' learning. Computers \& Education. 122: 243-259

Wollscheid, S., Sjaastad, J. \& Tømte C. (2016). The impact of digital devices vs. Pen(cil) and paper on primary school students' writing skills - A research review. Computers \& Education. 95:19-35

Veenman, M. V. J., Van Hout-Wolters, B. H. A. M. \& Afflerbach, P. (2006) Metacognition and learning: conceptual and methodological considerations. Metacognition Learning. 1: 3-14 